Functional Analysis and Its Applications, Vol. 40, No. 4, pp. 249-263, 2006

Translated from Funktsional'nyi Analiz i Ego Prilozheniya, Vol. 40, No. 4, pp. 3-21, 2006

Original Russian Text Copyright (c) by A. D. Baranov and V. P. Havin

\title{
Admissible Majorants for Model Subspaces, and Arguments of Inner Functions*
}

\author{
A. D. Baranov and V. P. Havin
}

Dedicated to the memory of Boris Yakovlevich Levin

Received March 15, 2006

\begin{abstract}
Let $\Theta$ be an inner function in the upper half-plane $\mathbb{C}^{+}$and let $K_{\Theta}$ denote the model subspace $H^{2} \ominus \Theta H^{2}$ of the Hardy space $H^{2}=H^{2}\left(\mathbb{C}^{+}\right)$. A nonnegative function $w$ on the real line is said to be an admissible majorant for $K_{\Theta}$ if there exists a nonzero function $f \in K_{\Theta}$ such that $|f| \leqslant w$ a.e. on $\mathbb{R}$. We prove a refined version of the parametrization formula for $K_{\Theta}$-admissible majorants and simplify the admissibility criterion (in terms of $\arg \Theta$ ) obtained in [8]. We show that, for every inner function $\Theta$, there exist minimal $K_{\Theta}$-admissible majorants. The relationship between admissibility and some weighted approximation problems is considered.
\end{abstract}

KEY WORDS: Hardy space, inner function, model subspace, entire function, Beurling-Malliavin theorem.

\section{Introduction and Main Results}

1.1. Let $\Theta$ be an inner function in the upper half-plane $\mathbb{C}^{+}$, that is, a bounded analytic function in $\mathbb{C}^{+}$such that $\lim _{y \rightarrow 0+}|\Theta(x+i y)|=1$ for almost all $x \in \mathbb{R}$ with respect to the Lebesgue measure on the real line $\mathbb{R}$.

With an inner function $\Theta$, we associate the model subspace

$$
K_{\Theta}=H^{2} \ominus \Theta H^{2}
$$

of the Hardy space $H^{2}=H^{2}\left(\mathbb{C}^{+}\right)$in the upper half-plane. These subspaces (and their analogs for the unit disk) play an outstanding role in both function and operator theories (see [6], [16]), in particular, in the Sz.-Nagy-Foias model for contractions of a Hilbert space.

Let us mention some concrete examples of model subspaces. If $\Theta(z)=\exp (i a z), a>0$, then $K_{\Theta}=\exp (i a z / 2) P W_{a / 2}$, where $P W_{a}$ stands for the Paley-Wiener space of entire functions of exponential type not exceeding $a$ that are square summable on $\mathbb{R}$.

On the other hand, if $B$ is a Blaschke product with zeros $z_{n}$ of multiplicities $m_{n}, n=1,2, \ldots$, that is,

$$
B(z)=\prod_{n} e^{i \alpha_{n}}\left(\frac{z-z_{n}}{z-\bar{z}_{n}}\right)^{m_{n}}
$$

(here $\alpha_{n} \in \mathbb{R}$ and the factors $e^{i \alpha_{n}}$ ensure the convergence of the product), then $K_{B}$ coincides with the closure in $L^{2}(\mathbb{R})$ of the linear span of the fractions $\left(z-\bar{z}_{n}\right)^{-l}, 1 \leqslant l \leqslant m_{n}, n=1,2, \ldots$.

Another important example is connected with de Branges' Hilbert spaces of entire functions (see [5]). Let the inner function $\Theta$ be meromorphic throughout the complex plane and let $z_{n}$ be the zeros of $\Theta$, counting multiplicities. Then there exists an entire function $E$ with zeros at $\bar{z}_{n}$ such that $\Theta=E^{*} / E$ and, consequently,

$$
|E(z)|>|E(\bar{z})|
$$

for $z \in \mathbb{C}^{+}$. Here $\left.E^{*}(z)=\overline{E(\bar{z})}\right)$. An entire function satisfying the above inequality is said to belong to the Hermite-Biehler class (we write $E \in H B$ ).

${ }^{*}$ The work is partially supported by RFBR grants No. 03-01-00377 and No. 06-01-00313. 
With every function $E \in H B$, we associate the de Branges space $\mathscr{H}(E)$ consisting of all entire functions $F$ such that $F / E$ and $F^{*} / E$ (restricted to $\mathbb{C}^{+}$) belong to the Hardy class $H^{2}=H^{2}\left(\mathbb{C}^{+}\right.$). It is easy to see that the mapping $F \mapsto F / E$ is a unitary operator from $\mathscr{H}(E)$ onto $K_{E^{*} / E}$, that is, $K_{E^{*} / E}=\mathscr{H}(E) / E$ (e.g., see [8, Theorem 3.1]).

1.2. We call a nonnegative function $w$ on $\mathbb{R}$ a majorant. A majorant $w$ is said to be admissible for $K_{\Theta}$ if there exists a nonzero function $f \in K_{\Theta}$ such that $|f(x)| \leqslant w(x)$ a.e. on $\mathbb{R}$. We denote the set of all admissible majorants for $K_{\Theta}$ by $\operatorname{Adm}(\Theta)$. An obvious necessary admissibility condition is the convergence of the logarithmic integral,

$$
\mathscr{L}(w)=\int_{\mathbb{R}} \log ^{+} w^{-1} d \Pi<\infty,
$$

where $\log ^{+} t=\max (\log t, 0)$ and $\Pi$ denotes the Poisson measure on $\mathbb{R}, d \Pi(t)=\pi^{-1}\left(1+t^{2}\right)^{-1} d t$. Indeed, as is well known, $\int_{\mathbb{R}} \log |f| d \Pi>-\infty$ for any nonzero $f \in H^{2}$. In Subsec. 5.1, we show that this necessary condition is never (i.e. for no $\Theta$ ) sufficient.

This fact is especially interesting for $\Theta(z)=\exp (i a z), a>0$. It gives rise to the well-known problem of describing admissible majorants for the Paley-Wiener space. In this important particular case, a wide class of admissible majorants is given by the famous Beurling-Malliavin theorem [4] asserting that if $\mathscr{L}(w)<\infty$ and

$$
\Omega=-\log w
$$

is a Lipschitz function on $\mathbb{R}$, then $w$ is an admissible majorant for any space $P W_{a}, a>0$. This is one of the deepest results of harmonic analysis, and a number of its different proofs is known (see [7], [12], [13] and the references therein).

Admissible majorants for general model subspaces of $H^{2}$ were studied for the first time by V. P. Havin and J. Mashreghi [8], [9]. Their approach is based on the study of the Hilbert transform of the function $\Omega$. Later this approach (in paper [14] by the same authors and F. L. Nazarov) led to a new (and, probably, the shortest) proof of the Beurling-Malliavin theorem. In [8], [9], a parametrization of $\operatorname{Adm}(\Theta)$ was found and a number of sufficient conditions for admissibility were obtained.

1.3. In the present paper, we obtain an essential refinement in the results of [8] and [9] and, in particular, give a new and simplified parametrization formula for $\operatorname{Adm}(\Theta)$. As a consequence, we show that, for any $\Theta$, there exist minimal admissible majorants (the definition of a minimal majorant is given in Subsec. 1.4).

We start with the general admissibility criterion [8]. Recall that the Hilbert transform of a function $g \in L^{1}(\Pi)$ is defined as

$$
\tilde{g}(x)=\mathrm{v} \cdot \mathrm{p} \cdot \frac{1}{\pi} \int_{\mathbb{R}}\left(\frac{1}{x-t}+\frac{t}{t^{2}+1}\right) g(t) d t .
$$

In what follows, we denote by $\arg \Theta$ the principal branch of the argument of the inner function $\Theta$, that is, $\arg \Theta \in(-\pi, \pi]$. Thus, $\arg \Theta$ is a measurable function defined a.e. on $\mathbb{R}$. If $\Theta$ is meromorphic, then it is more convenient to deal with a continuous branch of the argument. Note that each meromorphic inner function $\Theta$ is of the form

$$
\Theta(z)=\exp (i a z) B(z),
$$

where $a \geqslant 0$ and $B$ is a Blaschke product with zeros tending to infinity. In this case, there exists an increasing $C^{\infty}$-function $\varphi$ such that $\Theta(t)=\exp (i \varphi(t)), t \in \mathbb{R} ; \varphi$ is unique up to an additive constant $2 \pi k, k \in \mathbb{Z}$, and

$$
\varphi^{\prime}(t)=\left|\Theta^{\prime}(t)\right|=a+2 \sum_{n} \frac{m_{n} \operatorname{Im} z_{n}}{\left|t-z_{n}\right|^{2}},
$$

where $z_{n}$ are the zeros of $B$ with multiplicities $m_{n}$. We shall keep the notation $\varphi$ for the continuous argument of a meromorphic inner function $\Theta$.

We now state the admissibility criterion of Havin and Mashreghi [8], [9]. (Recall that $\Omega=$ $-\log w$. 
Theorem 1.1. A nonnegative function $w$ with $\Omega \in L^{1}(\Pi)$ belongs to $\operatorname{Adm}(\Theta)$ if and only if there exists a nonnegative function $m \in L^{\infty}(\mathbb{R}), m w \in L^{2}(\mathbb{R})$ and $\log m \in L^{1}(\Pi)$, and an inner function I such that

$$
\arg \Theta+2 \widetilde{\Omega}=2 \widetilde{\log m}+\arg I+2 \pi k \text { a.e. on } \mathbb{R},
$$

where $k$ is a measurable integer-valued function on $\mathbb{R}$.

For a given $\Theta,(1)$ can be regarded as a parametrization of $\operatorname{Adm}(\Theta)$. The parameters are the functions $m, k$, and $I$.

In Sec. 2, we shall show that the functional parameter $I$ can be replaced by a parameter $\gamma \in \mathbb{R}$, and get simplified parametrization formula for $\operatorname{Adm}(\Theta)$

Theorem 1.2. Let $w \geqslant 0$ and $\Omega \in L^{1}(\Pi)$. Then $w \in \operatorname{Adm}(\Theta)$ if and only if

$$
\arg \Theta+2 \widetilde{\Omega}=2 \widetilde{\log m}+2 \pi k+\gamma \quad \text { a.e. on } \mathbb{R},
$$

for some $m$ and $k$ with the same properties as above and for some constant $\gamma \in \mathbb{R}$.

This simplified parametrization follows immediately from (1) and from the fact that the argument of an arbitrary inner function can be represented as

$$
\arg I=2 \widetilde{\log m_{1}}+2 \pi k_{1}+\gamma \quad \text { a.e. on } \mathbb{R},
$$

where $\gamma \in \mathbb{R}, m_{1} \in L^{\infty}(\mathbb{R}) \cap L^{2}(\mathbb{R}), m_{1} \geqslant 0, \log m_{1} \in L^{1}(\Pi)$, and $k_{1}$ is a measurable integer-valued function.

In what follows, it will be meant that representations of the form (1)-(3) are true a.e. on $\mathbb{R}$.

1.4. Representation (3) has another interesting consequence. A $K_{\Theta}$-admissible majorant $w$ is said to be minimal if, for any $w \in \operatorname{Adm}(\Theta)$ such that $w_{1} \leqslant C w$ a.e. on $\mathbb{R}$, we have $w_{1} \asymp w$, that is, $c w \leqslant w_{1} \leqslant C w$ a.e., where $c$ and $C$ are positive constants.

Corollary 1.3. For each inner function $\Theta$, there is a minimal majorant $w \in \operatorname{Adm}(\Theta)$.

We prove this result in Sec. 5 and compare it with the results in [8] on the existence of minimal majorants (and, in particular, nonvanishing majorants; see Theorem 5.6). We obtain two different criteria for minimality (Propositions 5.4 and 5.6).

1.5. To apply Theorem 1.2, we need a description of functions $f$ which admit representation $f=2 \widetilde{\log m}+2 \pi k$ for some functions $m$ and $k$ with the above properties (see Theorem 1.1). We shall use the notion of a mainly increasing function introduced in [9].

Let $\left\{d_{n}\right\}$ be an increasing sequence of real numbers. We assume that either $n \in \mathbb{Z}$ and $\lim _{|n| \rightarrow \infty}\left|d_{n}\right|=\infty$ or $n \in \mathbb{N}$ and $\lim _{n \rightarrow \infty} d_{n}=\infty$; in the latter case we set $d_{0}=-\infty$. Let $I_{n}=\left(d_{n}, d_{n+1}\right)$. We denote by $\operatorname{Osc}(f, I)$ the oscillation of a function $f$ on the set $I$, that is, $\operatorname{Osc}(f, I)=\sup _{s, t \in I}(f(s)-f(t))$.

An absolutely continuous function $f$ on $\mathbb{R}$ is said to be mainly increasing if there exists an increasing sequence $\left\{d_{n}\right\}$ as above such that $f\left(d_{n+1}\right)-f\left(d_{n}\right) \asymp 1, n \in \mathbb{Z}(n \in \mathbb{N})$, and there is a constant $C>0$ such that

$$
\operatorname{Osc}\left(f, I_{n}\right) \leqslant C, \quad n \in \mathbb{Z}\left(\text { or } n \in \mathbb{Z}_{+}\right),
$$

and

$$
\frac{1}{\left|I_{n}\right|} \int_{I_{n}}\left|f^{\prime}(x)-f^{\prime}(t)\right| d t \leqslant C
$$

for almost all $x \in I_{n}$ and for all $n \in \mathbb{Z}$ (or $n \in \mathbb{N}$ ). By $|I|$, we denote the length of the interval $I$. In the case of one-sided sequences $\left\{d_{n}\right\}$, we assume that $f$ is a Lipschitz function on $\left(-\infty, d_{1}\right)$. Note that if $f \in C^{1}(\mathbb{R})$, then the integral condition (5) is implied by

$$
\operatorname{Osc}\left(f^{\prime}, I_{n}\right) \leqslant C, \quad n \in \mathbb{Z}\left(\text { or } n \in \mathbb{Z}_{+}\right)
$$

The following theorem gives a sufficient condition for a function $f$ to be representable modulo $2 \pi$ as a Hilbert transform of the logarithm of a bounded function. 
Theorem 1.4. Let $f$ be a mainly increasing function. Then $f$ admits the representation $f=$ $2 \widetilde{\log m}+2 \pi k+\gamma$ a.e on $\mathbb{R}$, where $m \in L^{\infty}(\mathbb{R}) \cap L^{2}(\mathbb{R}), m \geqslant 0, \log m \in L^{1}(\Pi), \gamma \in \mathbb{R}$, and $k$ is a measurable integer-valued function.

This theorem was proved in [9] under an additional restriction on the distances $\left|I_{n}\right|=d_{n+1}-d_{n}$, namely,

$$
\sum_{d_{n} d_{n+1}>0} \frac{\left|I_{n}\right|^{2}}{d_{n} d_{n+1}}<\infty
$$

(it holds, e.g., if $\sup _{n}\left|I_{n}\right| \leqslant \infty$ ). Our Theorem 1.4 shows that this condition can be dropped.

1.6. Let us mention a corollary of Theorem 1.4, which is useful for applications (see [3] where $\operatorname{Adm}(B)$ with a meromorphic Blaschke product $B$ is studied in terms of the distribution of zeros).

Corollary 1.5. Let $\Theta_{1}$ and $\Theta_{2}$ be meromorphic inner functions with continuous arguments $\varphi_{1}$ and $\varphi_{2}$ respectively. If $\varphi_{1}-\varphi_{2}$ is mainly increasing, then $\operatorname{Adm}\left(\Theta_{2}\right) \subset \operatorname{Adm}\left(\Theta_{1}\right)$.

The following corollary of Theorem 1.4 shows that if two inner functions (not necessarily meromorphic) are sufficiently close (in some sense), then the corresponding classes of admissible majorants coincide (see also Proposition 4.2 and Example after it).

Corollary 1.6. Let $\Theta_{1}$ and $\Theta_{2}$ be inner functions and let $\psi_{1}$ and $\psi_{2}$ be some branches of the arguments of $\Theta_{1}$ and $\Theta_{2}$, respectively. If $\psi_{1}-\psi_{2} \in L^{1}(\Pi)$ and

$$
\left(\psi_{1}-\psi_{2}\right)^{\sim} \in L^{\infty}(\mathbb{R}),
$$

then $\operatorname{Adm}\left(\Theta_{1}\right)=\operatorname{Adm}\left(\Theta_{2}\right)$.

In [8] and [9], $\operatorname{Adm}(\Theta)$ is studied mainly for meromorphic functions $\Theta$. The structure of the class $\operatorname{Adm}(\Theta)$ is especially well understood in two model situations. The first one concerns almost linear growth of the argument $\varphi$ of $\Theta$, that is, $\varphi^{\prime}(t) \asymp 1, t \in \mathbb{R}$ (see [9]). In this case, the class of admissible majorants coincides essentially with the class of admissible majorants for a PaleyWiener space, namely, it follows from Corollary 1.5 that $\operatorname{Adm}\left(e^{i a z}\right) \subset \operatorname{Adm}(\Theta) \subset \operatorname{Adm}\left(e^{i b z}\right)$ for some $b>a>0$ (see Sec. 4). The other case concerns the Blaschke products with zeros sufficiently sparse near the real axis (e.g., with pure imaginary zeros). In this situation, there exists a positive and continuous minimal majorant in $\operatorname{Adm}(\Theta)$, which, in a sense, is unique (see the discussion in Sec. 5).

In Sec. 6, we establish a relationship between admissibility problems for majorants and some problems of weighted approximation (see Proposition 6.1).

\section{Parametrization of Admissible Majorants}

We shall need an equivalent definition of the space $K_{\Theta}$, namely, a function $f$ belongs to $K_{\Theta}$ if and only if both $f$ and $\Theta \bar{f}$ are in $H^{2}$. (We consider $\Theta \bar{f}$ as a function on $\mathbb{R}$ and identify $H^{2}$-functions with their nontangential boundary traces on $\mathbb{R}$.)

Recall that the function

$$
\mathscr{K}_{z}(\zeta)=\frac{i}{2 \pi} \cdot \frac{1-\overline{\Theta(z)} \Theta(\zeta)}{\zeta-\bar{z}}
$$

is the reproducing kernel of $K_{\Theta}$ corresponding to the point $z \in \mathbb{C}^{+}$, that is, $\mathscr{K}_{z} \in K_{\Theta}$ and

$$
f(z)=\left\langle f, \mathscr{K}_{z}\right\rangle_{L^{2}(\mathbb{R})}, \quad f \in K_{\Theta} .
$$

This remains true if $z=x \in \mathbb{R}$ and $\Theta$ is analytic in a neighborhood of $x$.

With a nonnegative function $h$ such that $\log h \in L^{1}(\Pi)$, we associate the outer function $O_{h}$ with the modulus equal to $h$ a.e. on $\mathbb{R}$,

$$
O_{h}(z)=\exp \left(\frac{i}{\pi} \int_{\mathbb{R}}\left(\frac{1}{z-t}+\frac{t}{t^{2}+1}\right) \log h(t) d t\right), \quad z \in \mathbb{C}^{+} .
$$

Note that $O_{h}=h \exp (i \widetilde{\log h})$ a.e. on $\mathbb{R}$.

The following result on arguments of inner functions immediately implies Theorem 1.2. 
Theorem 2.1. Let $\Theta$ be an arbitrary inner function. Then there exists a function $m \in L^{\infty}(\mathbb{R}) \cap$ $L^{2}(\mathbb{R})$ with $m \geqslant 0$ and $\log m \in L^{1}(\Pi), \gamma \in \mathbb{R}$, and an integer-valued function $k$ such that

$$
\arg \Theta=2 \widetilde{\log m}+2 \pi k+\gamma \quad \text { a.e. on } \mathbb{R} \text {. }
$$

Remark. Representation (6) is equivalent to

$$
\Theta=e^{i \gamma} \frac{O_{m}}{\overline{O_{m}}} \text { a.e. on } \mathbb{R} .
$$

Representations of the form (7) appear in many problems of function theory (see [15]-[17] and the references therein; in [15], the right side of (7) is even said to be "ubiquitous"). A standard way to represent an inner function $\Theta$ in the form (7) is to write

$$
\Theta=-\bar{\alpha} \frac{\alpha-\Theta}{\bar{\alpha}-\bar{\Theta}} \quad \text { a.e. on } \mathbb{R}
$$

where $\alpha \in \mathbb{C},|\alpha|=1$. However, the bounded outer function $\alpha-\Theta$ is not necessarily in $H^{2}$, whereas, in what follows, we shall need a bounded and square summable $m$.

Proof of Theorem 2.1. It suffices to construct a bounded outer function $f$ in $K_{\Theta}$ such that $\bar{f} \Theta$ is also an outer function. Then $\bar{f} \Theta=e^{i \gamma} O_{|f|}$, whence

$$
\arg \Theta=2 \widetilde{\log |f|}+2 \pi k+\gamma
$$

It remains to set $m=|f|$.

We now construct a function $f$ with the desired properties. Let us assume that there is an $x_{0} \in \mathbb{R}$ such that $\Theta$ is analytic in the disk $\left|z-x_{0}\right|<1$. We set

$$
f(z)=-2 \pi i \mathscr{K}_{x_{0}}(z)=\frac{1-\overline{\Theta\left(x_{0}\right)} \Theta(z)}{z-x_{0}}, \quad z \in \mathbb{C}^{+} .
$$

Then $f \in K_{\Theta} \cap L^{\infty}(\mathbb{R})$. Note that $\operatorname{Re}\left(1-\overline{\Theta\left(x_{0}\right)} \Theta\right)>0$ in $\mathbb{C}^{+}$. As is known, in this case, $1-\overline{\Theta\left(x_{0}\right)} \Theta$ is an outer function, and therefore $f$ is also an outer function. Finally,

$$
\overline{f(t)} \Theta(t)=\frac{1-\Theta\left(x_{0}\right) \overline{\Theta(t)}}{t-x_{0}} \Theta(t)=-\Theta\left(x_{0}\right) f(t)
$$

a.e. on $\mathbb{R}$. Hence $\bar{f} \Theta$ is an outer function. Thus, the theorem is proved whenever there is a real point $x_{0}$ with the above property.

In the general case, we can represent the function $\Theta$ as a product of two inner functions $\Theta_{1}$ and $\Theta_{2}$ with singular spectra in $(-\infty, 0]$ and $[0, \infty)$ and zeros in $\{\operatorname{Re} z \leqslant 0\}$ and $\{\operatorname{Re} z \geqslant 0\}$, respectively. Hence, $\Theta_{1}$ is analytic in $\{\operatorname{Re} z<0\}$, whereas $\Theta_{2}$ is analytic in $\{\operatorname{Re} z>0\}$. Applying the above argument to $\Theta_{1}$ and $\Theta_{2}$, we get

$$
\arg \Theta_{j}=2 \widetilde{\log m_{j}}+2 \pi k_{j}+\gamma_{j}
$$

with $m_{j} \in L^{\infty}(\mathbb{R}) \cap L^{2}(\mathbb{R}), m_{j} \geqslant 0, \log m_{j} \in L^{1}(\Pi), j=1,2$. Adding these two relations together, we arrive at $(6)$.

Proof of Theorem 1.2. By Theorem 1.1, representation (2) implies the relation $w \in \operatorname{Adm}(\Theta)$. To prove the necessity, note that, by the same theorem, each $w \in \operatorname{Adm}(\Theta)$ satisfies

$$
\arg \Theta+2 \widetilde{\Omega}=2 \widetilde{\log m}+\arg I+2 \pi k
$$

for some $m, k$, and $I$. By Theorem 2.1,

$$
\arg I=2 \widetilde{\log m_{1}}+2 \pi k_{1}+\gamma,
$$

where $m_{1} \in L^{\infty}(\mathbb{R}) \cap L^{2}(\mathbb{R}), \log m_{1} \in L^{1}(\Pi), k_{1}$ is an integer-valued function, and $\gamma \in \mathbb{R}$. Hence,

$$
\arg \Theta+2 \widetilde{\Omega}=2 \widetilde{\log \left(m m_{1}\right)}+2 \pi\left(k+k_{1}\right)+\gamma,
$$

where $m m_{1} \in L^{\infty}(\mathbb{R}), m m_{1} w \in L^{2}(\mathbb{R})$, and $\log m m_{1} \in L^{1}(\Pi)$. 


\section{Mainly Increasing Functions. Proof of Theorem 1.4}

3.1. We start the proof of Theorem 1.4 with the following elementary lemma (giving a somewhat different definition of mainly increasing functions, which is needed for Theorem 1.4). We omit the proof.

Lemma 3.1. Let $f$ be a mainly increasing function. Then there exists an increasing sequence $\left\{d_{n}\right\}, n \in \mathbb{Z}$ or $n \in \mathbb{N}$, satisfying conditions (4), (5), and $f\left(d_{n}\right) \equiv 2 \pi n$.

In what follows we can assume without loss of generality that $d_{1}>0$, whereas $d_{0}<0$. We set $I_{n}=\left(d_{n}, d_{n+1}\right), l_{n}=\left(d_{n+1}-d_{n}\right) / 2$, and $c_{n}=\left(d_{n}+d_{n+1}\right) / 2, n \in \mathbb{Z}$ or $n \in \mathbb{N}$ in the case of one-sided sequences.

First note that if a function $g \in L^{1}(\Pi)$ is sufficiently smooth in a neighborhood of the origin and $g(0)=0$, then its Hilbert transform $\tilde{g}$ differs from its modified Hilbert transform $\check{g}$ by a constant,

$$
\check{g}(x)=\mathrm{v} \cdot \mathrm{p} \cdot \frac{1}{\pi} \int_{\mathbb{R}}\left(\frac{1}{x-t}+\frac{1}{t}\right) g(t) d t .
$$

And if $g$ vanishes identically outside a bounded interval, then $\check{g}$ coincides with $\mathscr{C}(g)$ up to an additive constant, where

$$
\mathscr{C}(g)(x)=\mathrm{v} \cdot \mathrm{p} \cdot \frac{1}{\pi} \int_{\mathbb{R}} \frac{g(t)}{x-t} d t .
$$

In the proof of Theorem 1.4, we shall use an auxiliary "saw-tooth" function $U$ with jumps at the points $d_{n}$. To define it, we start with a solitary "tooth" $u$, i.e. we set $u(x)=\pi x \chi_{(-1,1)}(x)\left(\chi_{E}\right.$ is the characteristic function of the set $E$ ). Consider the function

$$
F(x)=\mathscr{C}(u)(x)=-2+x \log \left|\frac{x+1}{x-1}\right|, \quad x \neq \pm 1 .
$$

It is easy to see that $F$ is even, bounded below by -2 , and $F(x)=\sum_{k=1}^{\infty} \frac{1}{(2 k+1) x^{2 k}}$ for $|x|>1$, so that $\left.F\right|_{(1,+\infty)}$ and $\left.F\right|_{(-\infty,-1)}$ are, respectively, decreasing and increasing.

Clearly, $\mathscr{C}$ commutes with shifts and multiplications of the independent variable by positive constants. Therefore, setting

$$
u_{n}(x)=u\left(\frac{x-c_{n}}{l_{n}}\right)=\frac{x-c_{n}}{l_{n}} \chi_{I_{n}}(x)
$$

we get

$$
\mathscr{C}\left(u_{n}\right)(x)=F\left(\frac{x-c_{n}}{l_{n}}\right)
$$

and

$$
\check{u}_{n}(x)=\mathscr{C}\left(u_{n}\right)(x)-\mathscr{C}\left(u_{n}\right)(0)=F\left(\frac{x-c_{n}}{l_{n}}\right)-F\left(\frac{c_{n}}{l_{n}}\right)
$$

Nowset

$$
U=\sum_{n \in \mathbb{Z}} u_{n}=u_{0}+U_{1}
$$

For a one-sided sequence $\left\{d_{n}\right\}$, we set $U(x)=0, x<d_{1}$. Clearly, $U$ is bounded on $\mathbb{R}$, whence $\widetilde{U}$ exists, and we have

$$
\widetilde{U}=\tilde{u}_{0}+\widetilde{U}_{1}=\tilde{u}_{0}+\check{U}_{1}+C_{1}=\mathscr{C}\left(u_{0}\right)+\check{U}_{1}+C_{2}=\mathscr{C}\left(u_{0}\right)+\sum_{n \neq 0} \check{u}_{n}+C_{2}
$$

(the last relation follows from the Lebesgue dominated convergence theorem).

3.2. The following lemma plays the key role in the proof of Theorem 1.4.

Lemma 3.2. There exist positive constants $A$ and $B$ such that

$$
\widetilde{U}(x) \geqslant-A-B \log (|x|+1), \quad x \in \mathbb{R} .
$$


Proof. We consider in detail the case of two-sided sequences. The proof for one-sided sequences is analogous. Without loss of generality, we assume that $x>0$.

The series in (9) converges uniformly on every compact interval in $I_{n}, n \in \mathbb{Z}$, whence $\widetilde{U}$ is continuous on $\bigcup_{n \in \mathbb{Z}} I_{n}$, and $\lim _{x \rightarrow d_{n}} \widetilde{U}(x)=+\infty$ for any $n \in \mathbb{Z}$. Thus, $\widetilde{U}$ is bounded below on any bounded interval, and it only remains to estimate $\widetilde{U}(x)$ for sufficiently large $x$. Fix $x>d_{1}, x \neq d_{n}$, $n \in \mathbb{N}$, and assume that $x \in I_{k}$. We can also assume that $d_{k}>e$. Then

$$
\begin{aligned}
\widetilde{U}(x) & =\check{u}_{k}(x)+\sum_{n: c_{n} \geqslant x / 2} \check{u}_{n}(x)+\sum_{n: c_{1} \leqslant c_{n}<x / 2} \check{u}_{n}(x)+\left(\mathscr{C}\left(u_{0}\right)(x)+\sum_{n \leqslant-1} \check{u}_{n}(x)\right)+C_{2} \\
& =\check{u}_{k}(x)+\sigma_{1}+\sigma_{2}+\sigma_{3}+C_{2} .
\end{aligned}
$$

We estimate $\check{u}_{k}(x)$ and $\sigma_{j}, j=1,2,3$, separately; but we begin with an estimate for $F\left(c_{n} / l_{n}\right)$. Note that $\left|c_{n}\right|>l_{n}, n \neq 0$, whence

$$
\begin{aligned}
F\left(\frac{c_{n}}{l_{n}}\right) & =\sum_{j=1}^{\infty} \frac{1}{2 j+1}\left(\frac{l_{n}}{c_{n}}\right)^{2 j}<\sum_{j=1}^{\infty} \frac{1}{j}\left(\frac{l_{n}}{c_{n}}\right)^{2 j} \\
& =-\log \left(1-\frac{l_{n}^{2}}{c_{n}^{2}}\right)=\log \frac{c_{n}^{2}}{d_{n} d_{n+1}}<\log \frac{d_{n+1}}{d_{n}},
\end{aligned}
$$

since $c_{n}<d_{n+1}$.

Estimate for $\check{\boldsymbol{u}}_{\boldsymbol{k}}(\boldsymbol{x})$. By (11),

$$
F\left(\frac{c_{k}}{l_{k}}\right)<\log \frac{d_{k+1}}{d_{k}}<\log d_{k+1}<\log 2 x
$$

if $x$ is in the right half of $I_{k}$, that is, $x \geqslant c_{k}$. In this case,

$$
\check{u}_{k}(x)=F\left(\frac{x-c_{k}}{l_{k}}\right)-F\left(\frac{c_{k}}{l_{k}}\right)>-2-\log 2-\log x
$$

since $F>-2$ everywhere. Suppose that $x \in I_{k}$ is to the left of $c_{k}$, that is, $d_{k}<x<c_{k}$. In this case, we consider two possibilities, (a) $c_{k} / l_{k}>2$ and (b) $c_{k} / l_{k} \leqslant 2$. Recall that $F$ is decreasing on $(1,+\infty)$, whence (a) implies $F\left(c_{k} / l_{k}\right)<F(2)$, and

$$
\check{u}_{k}(x)=F\left(\frac{x-c_{k}}{l_{k}}\right)-F\left(\frac{c_{k}}{l_{k}}\right)>-2-F(2) .
$$

Case (b) is more subtle. If (b) holds, then $c_{k} \asymp l_{k} \asymp d_{k+1}$, or, more precisely, $l_{k}<c_{k}<2 l_{k}$ and $2 l_{k}<d_{k+1}=c_{k}+l_{k}<3 l_{k}$. Direct calculation now gives (see the formula for $F$ )

$$
\begin{aligned}
\check{u}_{k}(x) & =F\left(\frac{x-c_{k}}{l_{k}}\right)-F\left(\frac{c_{k}}{l_{k}}\right) \\
& =\frac{c_{k}}{l_{k}} \log \frac{d_{k+1}-x}{d_{k+1}}+\frac{c_{k}}{l_{k}} \log d_{k}-\frac{x}{l_{k}} \log \left(d_{k+1}-x\right)-\frac{c_{k}-x}{l_{k}} \log \left(x-d_{k}\right) .
\end{aligned}
$$

Let us estimate the expression in (12) term by term. Since $d_{k+1}-x>l_{k}$ and $d_{k+1}<3 l_{k}$, the first term is no less than $-2 \log 3$; the second term is positive; the third is greater than

$$
-\frac{x}{l_{k}} \log d_{k+1}>-\frac{x}{3 d_{k+1}} \log d_{k+1}>-\frac{x}{3 x} \log x=-\frac{\log x}{3}
$$

(recall that the function $t \mapsto t^{-1} \log t$ decreases on $[e,+\infty)$ ); and, finally, the fourth term is no less than $-\log x$. Combining all these estimates, we see that, in case (b),

$$
\check{u}_{k}(x)>-A-B \log x
$$

with certain absolute positive constants $A$ and $B$. 
Estimate for $\boldsymbol{\sigma}_{1}$. We are going to show that all terms in the sum $\sigma_{1}$ are just positive. Indeed, if $n \neq k$ (as is the case with $\sigma_{1}$ ), then $x \notin I_{n}$, and $\left|x-c_{n}\right|>l_{n}$. And if $c_{n} \geqslant x / 2$ and $c_{n} \leqslant x$, then $1<c_{n} / l_{n} \leqslant\left(x-c_{n}\right) / l_{n} ; F$ decreases on $(1,+\infty)$, and therefore

$$
\check{u}_{n}(x)=F\left(\frac{x-c_{n}}{l_{n}}\right)-F\left(\frac{c_{n}}{l_{n}}\right)>0 .
$$

If $c_{n}>x$, then $-c_{n} / l_{n}<\left(x-c_{n}\right) / l_{n}<-1,\left.F\right|_{(-\infty,-1)}$ is increasing, and hence

$$
\check{u}_{n}(x)=F\left(\frac{x-c_{n}}{l_{n}}\right)-F\left(-\frac{c_{n}}{l_{n}}\right)>0
$$

(recall that $F$ is even).

Estimate for $\sigma_{2}$. If $x / 2>c_{n}$, then $x-c_{n}>c_{n}>l_{n}$ and $F\left(\left(x-c_{n}\right) / l_{n}\right)>0$. We see that

$$
\sigma_{2}=\sum_{n: c_{1} \leqslant c_{n}<x / 2}\left(F\left(\frac{x-c_{n}}{l_{n}}\right)-F\left(\frac{c_{n}}{l_{n}}\right)\right)>-\sum_{n: c_{1} \leqslant c_{n}<x / 2} F\left(\frac{c_{n}}{l_{n}}\right) .
$$

Using (11) and setting $m=\max \left\{n: c_{n}<x / 2\right\}$, we obtain

$$
\sigma_{2} \geqslant-\sum_{n: c_{1} \leqslant c_{n}<x / 2}\left(\log d_{n+1}-\log d_{n}\right)=-\left(\log d_{m+1}-\log d_{1}\right)>-\log x+\log d_{1},
$$

since $2 c_{m}=d_{m}+d_{m+1}<x$.

Estimate for $\sigma_{3}$. We have

$$
\begin{aligned}
\sigma_{3} & =\mathscr{C}\left(u_{0}\right)(x)+\sum_{n \leqslant-1} \check{u}_{n}(x)=\mathscr{C}\left(u_{0}\right)(x)+\sum_{n \leqslant-1} \frac{x}{\pi} \int_{I_{n}} \frac{U(t)}{t(x-t)} d t \\
& =F\left(\frac{x-c_{0}}{l_{0}}\right)+\frac{x}{\pi} \int_{-\infty}^{d_{0}} \frac{U(t)}{t(x-t)} d t .
\end{aligned}
$$

The first term is no less than -2 and the second admits a two-sided estimate (recall that $|U| \leqslant \pi$ ), its modulus is no greater than

$$
x \int_{-\infty}^{d_{0}} \frac{d t}{|t|(x-t)} \leqslant \log x+C
$$

where $C$ depends only on $d_{0}$.

Adding estimates (13), (14), and (15) together, we get the desired result.

3.3. We now are able to prove Theorem 1.4. Clearly, (9) implies the following assertion.

Lemma 3.3. $\widetilde{U}(x)=-2 \log \left|x-d_{n}\right|+\psi_{n}(x)$, where $\psi_{n}$ is a continuous function in a neighborhood of $d_{n}$.

Proof of Theorem 1.4. Now let $f$ be a mainly increasing function. By Lemma 3.1, there exists a sequence $\left\{d_{n}\right\}, n \in \mathbb{Z}$ or $n \in \mathbb{N}$, such that $f\left(d_{n}\right)=2 \pi n$.

As above, let $U$ be the function associated with the sequence $\left\{d_{n}\right\}$. Set

$$
g=f-\sum_{n}(2 n+1) \pi \chi_{I_{n}} \quad \text { and } \quad \tau=g-U .
$$

We define $\tau$ at the points $d_{n}$ as $\tau\left(d_{n}\right)=0$. Then $\tau$ is a bounded continuous function on $\mathbb{R}$. Moreover, by Lemma 2.7 in [9], $\tau$ is a Lipschitz function on $\mathbb{R}$. Hence, by the well-known properties of the Hilbert transform, $\tilde{\tau}$ is continuous, and we have

$$
\tilde{\tau}(x)=O(\log |x|), \quad|x| \rightarrow \infty
$$

(e.g., see [9, Lemma 2.8]).

The above estimate and Lemma 3.2 imply the existence of positive constants $A$ and $B$ such that

$$
\tilde{g}(x) \geqslant-A-B \log (|x|+1), \quad x \in \mathbb{R} .
$$


Set

$$
m(x)=\frac{\exp \left(-\frac{1}{2} \tilde{g}(x)\right)}{\prod_{j=1}^{2 K+1}\left|x-d_{n_{j}}\right|},
$$

where $d_{n_{j}}$ are different elements of the sequence $\left\{d_{n}\right\}$ and $K \in \mathbb{N}$. Let us show that $m$ has the desired properties for a sufficiently large $K$. By Lemma 3.3, $m$ is continuous. Moreover, if $2 K>B / 2$, then $m(x)=O\left(|x|^{-1}\right), x \rightarrow \infty$. Thus, $m \in L^{\infty}(\mathbb{R}) \cap L^{2}(\mathbb{R})$. Also, $\log m \in L^{1}(\Pi)$, since $g \in L^{\infty}(\mathbb{R})$.

Let us evaluate the Hilbert transform of $\log m$. It is easy to see that $\widetilde{(\log |t|})(x)=-\frac{\pi}{2} \operatorname{sign} x+$ const, and, consequently,

$$
\left(\log \left|t-d_{n_{j}}\right|\right)^{\sim}(x)=-\frac{\pi}{2} \operatorname{sign}\left(x-d_{n_{j}}\right)+\gamma_{j}
$$

for a real constant $\gamma_{j}$. We have

$$
2 \widetilde{\log m}(x)=-\tilde{\tilde{g}}(x)+\pi \sum_{j=1}^{2 K+1} \operatorname{sign}\left(x-d_{n_{j}}\right)-2 \sum_{j=1}^{2 K+1} \gamma_{j} .
$$

We have $\tilde{\tilde{g}}=-g+\gamma_{0}$ for a constant $\gamma_{0}$, since $g \in L^{\infty}(\mathbb{R})$. Hence

$$
2 \widetilde{\log m}(x)=g(x)+\pi \sum_{j=1}^{2 K+1}\left(1+\operatorname{sign}\left(x-d_{n_{j}}\right)\right)-(2 K+1) \pi+\gamma,
$$

where $\gamma=-\gamma_{0}-2 \sum_{j=1}^{2 K+1} \gamma_{j}$. Consequently, for $x \neq d_{n}$,

$$
\begin{aligned}
f(x) & =g(x)-\pi \sum_{n}(2 n+1) \chi_{I_{n}}(x) \\
& =2 \widetilde{\log m}(x)-\pi \sum_{j=1}^{2 K+1}\left(1+\operatorname{sign}\left(x-d_{n_{j}}\right)\right)+\pi\left(\sum_{n}(2 n+1) \chi_{I_{n}}(x)-(2 K+1)\right)+\gamma .
\end{aligned}
$$

Thus, $f=2 \widetilde{\log m}+2 \pi k+\gamma$, where $k$ is an integer-valued function.

\section{Corollaries of Theorem 1.4. Majorization in the Mean}

4.1. In this section we prove Corollaries 1.5 and 1.6. We also consider a somewhat different majorization problem, namely, the admissibility with respect to $L^{p}$-norms.

Proof of Corollary 1.5. Let $w \in \operatorname{Adm}\left(\Theta_{2}\right)$. By Theorem 1.2,

$$
\varphi_{2}+2 \widetilde{\Omega}=2 \widetilde{\log m_{1}}+2 \pi k_{1}+\gamma_{1},
$$

where $m_{1} \geqslant 0, m_{1} \in L^{\infty}(\mathbb{R}), \log m_{1} \in L^{1}(\Pi), m_{1} w \in L^{2}(\mathbb{R}), k_{1}$ is an integer-valued function, and $\gamma_{1} \in \mathbb{R}$. On the other hand, by Theorem 1.4, the mainly increasing function $\varphi_{1}-\varphi_{2}$ admits the representation

$$
\varphi_{1}-\varphi_{2}=2 \widetilde{\log m_{2}}+2 \pi k_{2}+\gamma_{2} .
$$

Adding these two relations together, we get the desired representation for the function $\varphi_{1}+2 \widetilde{\Omega}$ (note that $m_{1} m_{2} w \in L^{2}(\mathbb{R})$ ).

We immediately get the following corollary concerning the inner functions with "almost linear" growth of the argument.

Corollary 4.1. Let $\Theta$ be a meromorphic inner function such that $\varphi^{\prime} \asymp 1$. Then there exist positive numbers $a$ and $b$ such that $\operatorname{Adm}\left(e^{i a z}\right) \subset \operatorname{Adm}(\Theta) \subset \operatorname{Adm}\left(e^{i b z}\right)$.

Proof. Note that $\psi(t)=b t$ is the continuous argument of the inner function $e^{i b z}$. Let $c \leqslant$ $\varphi^{\prime}(t) \leqslant C, t \in \mathbb{R}$, for some positive constants $c$ and $C$. If $b>C$, then $b t-\varphi(t)$ is an increasing Lipschitz function and, consequently, it is mainly increasing. By Corollary 1.5, $\operatorname{Adm}(\Theta) \subset \operatorname{Adm}\left(e^{i b z}\right)$. The proof of the second inclusion is analogous. 
Proof of Corollary 1.6. We use the following property of the Hilbert transform: if $g$ and $\tilde{g}$ are in $L^{1}(\Pi)$, then $\tilde{\tilde{g}}=-g+$ const. Now set $g=\left(\psi_{2}-\psi_{1}\right)^{\sim} / 2$ and $m_{1}=e^{-g}$. We have

$$
\psi_{2}-\psi_{1}=2 \widetilde{\log m_{1}}+\gamma_{1},
$$

and $m_{1} \asymp 1$, since, by the hypothesis, $g \in L^{\infty}(\mathbb{R})$.

Let $w \in \operatorname{Adm}\left(\Theta_{1}\right)$. Then, by Theorem 1.2,

$$
\psi_{1}+2 \widetilde{\Omega}=2 \widetilde{\log m}+2 \pi k+\gamma,
$$

where $m \in L^{\infty}(\mathbb{R}), m \geqslant 0, m w \in L^{2}(\mathbb{R}), \log m \in L^{1}(\Pi), \gamma \in \mathbb{R}$, and $k$ is an integer-valued function. Hence,

$$
\psi_{2}+2 \widetilde{\Omega}=2 \widetilde{\log \left(m m_{1}\right)}+2 \pi k+\gamma+\gamma_{1},
$$

and therefore $w \in \operatorname{Adm}\left(\Theta_{2}\right)$. The opposite inclusion is analogous.

The following proposition explains why the classes of admissible majorants in Corollary 1.6 coincide (for the proof, see [2]).

Proposition 4.2. Let $\Theta_{1}$ and $\Theta_{2}$ be meromorphic inner functions with continuous increasing branches of the arguments $\varphi_{1}$ and $\varphi_{2}$. Assume that $\varphi_{1}-\varphi_{2} \in L^{1}(\Pi)$ and $\left(\varphi_{1}-\varphi_{2}\right)^{\sim} \in L^{\infty}(\mathbb{R})$. Then there exist entire functions $E_{1}, E_{2} \in H B$ such that $\Theta_{1}=E_{1}^{*} / E_{1}, \Theta_{2}=E_{2}^{*} / E_{2}$ and $\left|E_{1}(z)\right| \asymp$ $\left|E_{2}(z)\right|, z \in \overline{\mathbb{C}^{+}}$. In particular, $\mathscr{H}\left(E_{1}\right)=\mathscr{H}\left(E_{2}\right)$, and the respective norms are equivalent.

Examples. 1. Let $\Theta_{1}(z)=e^{2 \pi i z}$ and let $\Theta_{2}$ be the Blaschke product with the zeros $z_{n}=n+i$, $n \in \mathbb{Z}$. Then $\Theta_{l}=E_{l}^{*} / E_{l}, l=1,2$, where $E_{1}(z)=\exp (-i \pi z)$ and $E_{2}(z)=\sin \pi(z+i)$. Clearly, $\left|E_{1}(z)\right| \asymp\left|E_{2}(z)\right|, z \in \overline{\mathbb{C}^{+}}$, and so $\operatorname{Adm}\left(\Theta_{1}\right)=\operatorname{Adm}\left(\Theta_{2}\right)$.

2. For an inner function $\Theta_{1}$ and for $\zeta \in \mathbb{C},|\zeta|<1$, one can consider the Frostman shift $\Theta_{2}$ of $\Theta_{1}$,

Then we have

$$
\Theta_{2}=\frac{\Theta_{1}-\zeta}{1-\bar{\zeta} \Theta_{1}} .
$$

$$
\frac{\Theta_{2}}{\Theta_{1}}=\frac{\overline{1-\bar{\zeta} \Theta_{1}}}{1-\bar{\zeta} \Theta_{1}}=\frac{\bar{h}}{h}
$$

a.e. on $\mathbb{R}$, where $h=1-\bar{\zeta} \Theta_{1}$ is an outer function in $H^{\infty}$ and $|h| \asymp 1$. Thus, $\psi_{1}-\psi_{2}=$ $2 \widetilde{\log |h|}$ for some choice of the arguments $\psi_{1}$ and $\psi_{2}$ for $\Theta_{1}$ and $\Theta_{2}$. By Corollary 1.6, $\operatorname{Adm}\left(\Theta_{1}\right)=$ $\operatorname{Adm}\left(\Theta_{2}\right)$.

4.2. We now consider a slightly different majorization problem, which can be called "majorization in the mean". We say that $w$ is an $L^{p}$-admissible majorant for $K_{\Theta}, p>0$, if there exists a nonzero $f \in K_{\Theta}$ such that $f / w \in L^{p}(\mathbb{R})$. In these terms, our "old" admissible majorants (see Subsec. 1.2) become $L^{\infty}$-admissible. It follows from the construction of $m$ in the proof of Theorem 1.4 (see (16)) that if $K$ is sufficiently large, then $m \in L^{p}(\mathbb{R})$ for any given $p>0$. Thus, we have

Theorem 4.3. If $f$ is mainly increasing, then, for any $p>0$, there exists an $m \geqslant 0$ with $\log m \in L^{1}(\Pi)$ and $m \in L^{\infty}(\mathbb{R}) \cap L^{p}(\mathbb{R}), \gamma \in \mathbb{R}$, and an integer-valued $k$ such that

$$
f=2 \widetilde{\log m}+2 \pi k+\gamma \quad \text { a.e. on } \mathbb{R} .
$$

Corollary 4.4. Let $\Theta$ be a meromorphic inner function. If $w \in L^{\infty}(\mathbb{R})$ and the function $\varphi+2 \widetilde{\Omega}$ is mainly increasing, then $w$ is an $L^{p}$-admissible majorant for $K_{\Theta}$ and for any $p>0$.

\section{Minimal Majorants}

5.1. In this section, we prove, as a consequence of Theorem 2.1, the existence of minimal admissible majorants. Recall that a majorant $w \in \operatorname{Adm}(\Theta)$ is said to be minimal if, for any $w_{1} \in \operatorname{Adm}(\Theta)$ such that $w_{1} \leqslant C w$, we have $w_{1} \asymp w$ a.e. on $\mathbb{R}$.

We say that an inner function $\Theta$ is the circular part of a function $g$ in the Hardy space $H^{1}$ if $g=\Theta|g|$ a.e. on $\mathbb{R}$. We shall need the following result in [8]. 
Theorem 5.1. If $\Theta$ is the circular part of an outer function $g \in H^{1}$, then $w=|g|^{1 / 2}$ is a minimal majorant for $K_{\Theta}$.

In [8], Theorem 5.1 was obtained as a consequence of a stronger result on minimality. Namely, if $g$ is an outer function (not necessarily in $H^{1}$ ) and the inner function $\Theta$ is its circular part, then any $w_{1} \in \operatorname{Adm}(\Theta)$ such that

$$
\int_{\mathbb{R}} w_{1}|g|^{-1 / 2} d \Pi<\infty
$$

satisfies $|g|^{1 / 2} \leqslant C w_{1}$ a.e. for some constant $C>0$ [8, Theorem 5.2]. Here we give a new and direct proof of Theorem 5.1 based on the following lemma.

Lemma 5.2. Let $m \geqslant 0, m \in L^{\infty}(\mathbb{R})$, and $\log m \in L^{1}(\Pi)$. If

$$
2 \widetilde{\log m}+2 \pi k+\gamma=0 \text { a.e. on } \mathbb{R},
$$

where $\gamma \in \mathbb{R}$ and $k$ is an integer-valued function, then $\operatorname{ess~}_{\inf } m>0$.

Proof. It follows from (17) and parametrization formula (2) that $m w \in \operatorname{Adm}(\Theta)$ for each inner function $\Theta$ and each $w \in \operatorname{Adm}(\Theta)$. Indeed, if

$$
\arg \Theta-2 \widetilde{\log w}=2 \widetilde{\log m_{1}}+2 \pi k_{1}+\gamma_{1},
$$

then

$$
\arg \Theta-2 \widetilde{\log m w}=2 \widetilde{\log m_{1}}+2 \pi\left(k+k_{1}\right)+\gamma+\gamma_{1}
$$

and therefore $m w \in \operatorname{Adm}(\Theta)$.

Set $\Theta(z)=(z-i) /(z+i)$. In this case, $K_{\Theta}$ is the one-dimensional space generated by the function $f(z)=(z+i)^{-1}$. Thus, the majorant $w(t)=|t+i|^{-1}$ is admissible. Since $m w \in \operatorname{Adm}(\Theta)$, we have $m(t) /|t+i| \geqslant \delta /|t+i|$ a.e. for a $\delta>0$, whence ess $\inf _{\mathbb{R}} m \geqslant \delta$.

Proof of Theorem 5.1. Let $g$ be an outer function in $H^{1}$ such that $\Theta|g|=g$, and, consequently, $\arg \Theta=\widehat{\log |g|}+2 \pi k+\gamma$ for an integer-valued function $k$. Put $h=|g|^{1 / 2}$ and let $O_{h}$ be the outer function with modulus $h$ on $\mathbb{R}$. Then

$$
\Theta \overline{O_{h}}=h \exp (i(\arg \Theta-\widetilde{\log h}))=h \exp (i(\widetilde{\log h}+2 \pi k+\gamma))=e^{i \gamma} O_{h} \text { a.e. on } \mathbb{R},
$$

and, thus, $O_{h} \in K_{\Theta}$. In particular, $h \in \operatorname{Adm}(\Theta)$.

Now let $m h \in \operatorname{Adm}(\Theta)$ where $m \in L^{\infty}(\mathbb{R}), m \geqslant 0$, and $\log m \in L^{1}(\Pi)$. Then, by (2),

$$
\arg \Theta-2 \widetilde{\log m h}=2 \widetilde{\log m_{1}}+2 \pi k_{1}+\gamma_{1} \text {. }
$$

Since $\arg \Theta=2 \widetilde{\log h}+2 \pi k+\gamma$, we have

$$
2 \widetilde{\log m}+2 \widetilde{\log m_{1}}+2 \pi\left(k+k_{1}\right)+\gamma+\gamma_{1}=0 .
$$

By Lemma $5.2, m m_{1} \geqslant \delta$ a.e. for some $\delta>0$. It follows that $m \geqslant \delta_{1}>0$ a.e. on $\mathbb{R}$ for some $\delta_{1}>0$, since $m_{1} \in L^{\infty}$.

Proof of Corollary 1.3. We now show that each inner function $\Theta$ is the circular part of some outer $H^{1}$-function. Therefore, $\operatorname{Adm}(\Theta)$ contains minimal majorants for an arbitrary inner function $\Theta$.

By Theorem 2.1, there is a nonnegative $m$ in $m \in L^{\infty}(\mathbb{R}) \cap L^{2}(\mathbb{R})$ with $\log m \in L^{1}(\Pi)$ such that

$$
\arg \Theta=2 \widetilde{\log m}+2 \pi k+\gamma .
$$

Set $g=e^{i \gamma} O_{m^{2}}$. Then $g$ is an outer function in $H^{1}$ and $\Theta|g|=g$. Thus, the conditions of Theorem 5.1 are fulfilled.

Example. Let $\Theta(z)=\exp (2 i a z), a>0$, and let $x_{0} \in \mathbb{R}$. Then $w(t)=\pi\left|\mathscr{K}_{x_{0}}(t)\right|=\left|\frac{\sin a\left(t-x_{0}\right)}{t-x_{0}}\right|$ is a minimal majorant for $K_{\Theta}$ (see the proof of Theorem 2.1).

It follows from Corollary 1.3 that, for each inner function $\Theta$, the class $\operatorname{Adm}(\Theta)$ is essentially smaller than the class of all $H^{2}$-majorants (that is, nonnegative $L^{2}$-functions with a finite logarithmic integral). 
Corollary 5.3. Let $\Theta$ be an inner function. Then there exists a function $w \in L^{2}(\mathbb{R})$ such that $\mathscr{L}(w)<\infty$ and $w \notin \operatorname{Adm}(\Theta)$.

Proof. By Corollary 1.3, there exists a minimal majorant $m_{0}$ for $K_{\Theta}$. Set $w_{0}(t)=(|t|+1)^{-1}$ and $w=w_{0} m_{0}$. Clearly, $\mathscr{L}(w)<\infty$, but $w \notin \operatorname{Adm}(\Theta)$. Indeed, $w \leqslant m_{0}$, but the inequality $m_{0} \leqslant C w$ does not hold, although $m_{0}$ is a minimal majorant in $\operatorname{Adm}(\Theta)$.

5.2. The following proposition gives a parametrization formula for minimal majorants.

Proposition 5.4. Let $w \geqslant 0$ and let $\log w \in L^{1}(\Pi)$. Then $w$ is a minimal $K_{\Theta}$-admissible majorant if and only if $w \in L^{2}(\mathbb{R})$ and there exists a function $w_{1} \asymp w$ and $a \gamma \in \mathbb{R}$ such that

$$
\Theta=e^{i \gamma} \frac{O_{w_{1}}}{\overline{O_{w_{1}}}} \quad \text { a.e. on } \mathbb{R} .
$$

Proof. Clearly, (18) implies $O_{w_{1}}^{2}=e^{-i \gamma} \Theta\left|O_{w_{1}}^{2}\right|$ and, therefore, $e^{-i \gamma} \Theta$ is the circular part of the $H^{1}$-function $O_{w_{1}}^{2}$. The minimality of $w_{1}$ (and of $w$ ) now follows from Theorem 5.1.

Conversely, assume that $w$ is a minimal majorant for $K_{\Theta}$. Then there exists a nonzero $f \in K_{\Theta}$ such that $|f| \leqslant w$ a.e. on $\mathbb{R}$. We have $w \asymp|f|$, since $w$ is minimal, and therefore $w \in L^{2}(\mathbb{R})$. By the admissibility criterion, we have

$$
\arg \Theta-2 \widetilde{\log m w}=2 \pi k+\gamma
$$

for some $m \in L^{\infty}(\mathbb{R}), m \geqslant 0, \log m \in L^{1}(\Pi)$. We have $m \asymp 1$, since $m w \leqslant C w$ and $w$ is minimal. Set $w_{1}=m w$. Then

which is equivalent to (18).

$$
\arg \Theta=2 \widetilde{\log w_{1}}+2 \pi k+\gamma
$$

Theorem 5.1 and Proposition 5.4 yield a parametrization for the set $\operatorname{Adm}_{*}(\Theta)$ of all minimal majorants in $\operatorname{Adm}(\Theta)$,

$$
\operatorname{Adm}_{*}(\Theta)=\left\{w \geqslant 0 \text { on } \mathbb{R}: w \asymp w_{1}, \log w_{1} \in L^{1}(\Pi) \text { and } \widetilde{\log w_{1}}=\frac{1}{2} \arg \Theta+\pi k+\gamma\right\},
$$

where $k$ is an integer-valued function and $\gamma \in \mathbb{R}$. Note that, for the whole class $\operatorname{Adm}(\Theta)$, we have an analogous description with one more parameter $\widetilde{\log m}$. Unfortunately, the parameter $k$ in (19) is not quite "free", namely, it must be of the form $\tilde{g}$ for a $g \in L^{1}(\Pi)$, since $\arg \Theta$ is bounded, and thus is of this form. For example, an integer-valued $k$ can only appear in (19) if

$$
\Pi(\{|k|>a\})=o(1 / a), \quad a \rightarrow \infty
$$

(according to the Kolmogorov theorem, see [11]).

5.3. We now discuss another approach to admissible majorants applicable to the case of meromorphic Blaschke products with sparse zeros. In this case the model subspaces are closely related to the de Branges spaces $\mathscr{H}(E)$ (see Introduction).

By Corollary 1.3, minimal majorants exist in any $\operatorname{Adm}(\Theta)$. However, such majorants can have zeros on the real axis (the majorants constructed in the proof of Theorem 2.1 vanish at some real points where $\Theta=1$ ). One can ask the following natural question: for what model subspaces are there "strictly positive" (i.e. separated from zero on any bounded interval) minimal majorants? It turns out that the relation $1 \in \mathscr{H}(E)$ is crucial for the existence of a positive minimal majorant. Namely, we have the following criterion.

Theorem 5.5. Let $E$ be an entire function of zero exponential type in the Hermite-Biehler class $H B$ and let $\Theta=E^{*} / E$. Then the following assertions are equivalent:

(1) $1 / E \in L^{2}(\mathbb{R})$;

(2) there exists a positive and continuous minimal majorant for $K_{\Theta}$.

In this case, $w=1 /|E|$ is a positive minimal majorant, and it is the unique continuous positive minimal majorant for $K_{\Theta}$ up to multiplication by a function $m \asymp 1$.

This theorem was proved in [8] under the additional assumption that the series $\sum_{n}\left|z_{n}\right|^{-1} \log \left|z_{n}\right|$ converges and in [1] under the weaker condition $\sum_{n}\left|z_{n}\right|^{-1}<\infty$. Here we give a new and very short 
proof of the implication $1 \Longrightarrow 2$ (for the proof of $2 \Longrightarrow 1$ and of the uniqueness of a positive and continuous minimal majorant, see [8]).

Proof of Theorem 5.5. Let $1 / E \in L^{2}(\mathbb{R})$. Since $E$ is a Hermite-Biehler function of order $\leqslant 1$, it belongs to the Polya class (see [5, Chap. 1, Sec. 7]), that is, $|E(x+i y)|$ is an increasing function of $y \geqslant 0$ for any $x \in \mathbb{R}$. Therefore the relation $1 / E \in L^{2}(\mathbb{R})$ implies the relation $1 / E \in H^{2}$. Also note that $\Theta / \bar{E}=1 / E$ on $\mathbb{R}$. Hence, $1 / E \in K_{\Theta}$ (for the related discussion, see the beginning of Sec. 2).

The function $1 / E$ can now be written for $\mathbb{C}^{+}$as $e^{i \gamma} e^{i a z} O_{1 /|E|}$ with some $\gamma \in \mathbb{R}$ and $a \geqslant 0$, since $1 / E$ is analytic on $\mathbb{R}$ and does not vanish in $\mathbb{C}^{+}$. The function $E$ is of minimal type, and therefore

$$
\limsup _{y \rightarrow \infty} \frac{\log |E(i y)|}{y}=0 .
$$

Hence, $a=0$, and, consequently, $1 / E$ and $1 / E^{2}$ are outer functions. The minimality of $1 /|E|$ now follows from Theorem 5.1, since $\Theta /|E|^{2}=1 / E^{2}$ on $\mathbb{R}$.

Remark. For a number of conditions stated in terms of zeros of $E$ and ensuring the relation $1 / E \in L^{2}(\mathbb{R})$, see [1] and [10].

5.4. We conclude with another description of minimal majorants in somewhat different terms. For a majorant $w \in \operatorname{Adm}(\Theta)$, we consider the set

$$
E_{w}(\Theta)=\left\{f \in K_{\Theta}:|f| \leqslant C w \text { a.e. on } \mathbb{R} \text { for some } C>0\right\} .
$$

Clearly, $E_{w}(\Theta)$ is a (generally, nonclosed) linear subspace of $K_{\Theta}$.

Proposition 5.6. Let $w \in \operatorname{Adm}(\Theta)$. Then $w$ is a minimal majorant for $K_{\Theta}$ if and only if the space $E_{w}(\Theta)$ is one-dimensional and $w \asymp|f|$ for a nonzero $f \in E_{w}(\Theta)$.

Proof. Let $E_{w}(\Theta)$ be a one-dimensional subspace of $K_{\Theta}$ and let $w \asymp|f|$, where $f$ is a nonzero function in $E_{w}(\Theta)$. If $w_{1} \leqslant C w$ a.e. and $w_{1} \in \operatorname{Adm}(\Theta)$, then there is a nonzero $g \in E_{w}(\Theta)$ such that $|g| \leqslant w_{1}$. Hence, $w \asymp|g|$, and thus, $w \leqslant C_{1} w_{1}$ a.e. on $\mathbb{R}$.

Now let $w$ be a minimal majorant. Then $w \asymp|f|$ for any nonzero $f \in K_{\Theta}$ such that $|f| \leqslant C w$ a.e. on $\mathbb{R}$. Assume that $\operatorname{dim} E_{w}(\Theta) \geqslant 2$. Then there exist linearly independent elements $f_{1}$ and $f_{2}$ in $K_{\Theta}$ such that $\left|f_{j}\right| \leqslant w$ a.e., $j=1,2$. We can choose constants $\alpha_{1}$ and $\alpha_{2},\left|\alpha_{1}\right|+\left|\alpha_{2}\right|>0$, such that $\alpha_{1} f_{1}\left(z_{0}\right)+\alpha_{2} f_{2}\left(z_{0}\right)=0$ at some point $z_{0} \in \mathbb{C}^{+}$. It is easy to see that

$$
f(z)=\frac{\alpha_{1} f_{1}(z)+\alpha_{2} f_{2}(z)}{z-z_{0}}
$$

is a nonzero function in $K_{\Theta}$ and $|f(t)| \leqslant C(1+|t|)^{-1} w(t)$ a.e. on $\mathbb{R}$, which contradicts the minimality of $w$.

Remarks. 1. We have in fact proved the following assertion on "exact" elements of Adm $(\Theta)$, i.e. majorants $|f|$ with $f$ a non-zero element of $K_{\Theta}:|f|$ is minimal if and only if $w \notin \operatorname{Adm}(\Theta)$, where $w(t)=|f(t)| /(1+|t|), t \in \mathbb{R}$. This follows from the fact that either $|f|$ is minimal or $\operatorname{dim} E_{|f|}(\Theta) \geqslant 2$.

2. The assumptions $w \in \operatorname{Adm}(\Theta)$ and $\operatorname{dim} E_{w}(\Theta)=1$ do not imply the minimality of $w$. For example, let $\Theta(z)=E^{*}(z) / E(z)$, where $E(z)=(z+i)^{2}$. Then $1 / E \in L^{2}(\mathbb{R})$ and, by Theorem 5.5, $w=1 /|E|$ is a minimal majorant for $K_{\Theta}$. Now set $w_{1}(t)=\sqrt{|t|+1} /|E(t)|$. Each element of $K_{\Theta}$ is of the form $P / E$ where $P$ is a polynomial of at most degree 1 . Hence, $E_{w}(\Theta)=E_{w_{1}}(\Theta)=\operatorname{Span}\{1 / E\}$. However, the majorant $w_{1}$ is not minimal.

The following criterion for minimality is an immediate consequence of Proposition 5.6

Corollary 5.7. A majorant $w \in \operatorname{Adm}(\Theta)$ is minimal for $K_{\Theta}$ if and only if there exists a function $h_{w} \in K_{\Theta}$ such that $\left|h_{w}\right| \asymp w$ and, for any $f \in K_{\Theta}$ satisfying $|f| \leqslant C w$ a.e., we have $f=\alpha h_{w}$ for some constant $\alpha \in \mathbb{C}$.

Examples. 1. Let $\Theta$ be a meromorphic inner function. For this case, we have already considered two examples of minimal majorants. If $E$ is of zero exponential type and $1 / E \in K_{\Theta}$, then $|E|^{-1}$ is 
the unique positive minimal majorant. On the other hand, we have seen in the proof of Theorem 2.1 that $w(t)=\left|\mathscr{K}_{s}(t)\right|, s \in \mathbb{R}$, is a minimal majorant for $K_{\Theta}$ (this assertion also follows from the de Branges theory; see [5, Theorem 22]).

These two situations are in a sense "extremal". The majorant $|E|^{-1}$ has the fastest possible decay and no real zeros. On the other hand, a majorant of the form $\left|\mathscr{K}_{s}\right|$ has many real zeros and slow decay. Between these two cases, there is a wide class of minimal majorants, where the slower decay is compensated for by a greater number of real zeros. The following two examples will illustrate what has been said.

2. Let $\Theta=\Theta_{1} \Theta_{2}$. If $w_{j}$ is a minimal majorant for $K_{\Theta_{j}}, j=1,2$, and $w_{1} w_{2} \in L^{2}(\mathbb{R})$, then, by Proposition 5.4, $w_{1} w_{2}$ is a minimal majorant for $K_{\Theta}$. Now let $\Theta_{j}$ be Blaschke product with imaginary zeros, let $E_{j}$ be the corresponding Hermite-Biehler entire function, and let $\mathscr{K}_{s}^{(j)}$ denote the reproducing kernel of the space $K_{\Theta_{j}}$. Then the majorants $\left|E_{1}\right|^{-1}\left|\mathscr{K}_{s}^{(2)}\right|$ and $\left|E_{2}\right|^{-1}\left|\mathscr{K}_{s}^{(1)}\right|$ are minimal for $K_{\Theta}$.

3. Let $B$ be a finite Blaschke product of degree $N$. Then $w$ is a minimal majorant for $K_{B}$ if and only if there is a polynomial $P$ of degree $n, 0 \leqslant n \leqslant N-1$, having real zeros and satisfying the relation

$$
w(t) \asymp|P(t)|(1+|t|)^{-N}
$$

and thus, $w(t) \asymp|t|^{n-N},|t| \rightarrow \infty$.

\section{Admissibility and Approximation}

In this section, we show that admissibility is equivalent to a certain approximation property of the majorant. For a nonnegative function $u$, we denote by $L^{q}(u)$ the space of functions $f$ on $\mathbb{R}$ such that $\int_{\mathbb{R}}|f|^{q} u<\infty$, and we set $H_{-}^{2}=L^{2}(\mathbb{R}) \ominus H^{2}$. Note that the space $H_{-}^{2} \oplus \Theta H^{2}$ is the orthogonal complement of $K_{\Theta}$ in $L^{2}(\mathbb{R})$.

Proposition 6.1. Let $2 \leqslant p \leqslant \infty$, let $q=p /(p-1)$, and let $w \in L^{2 p /(p-2)}(\mathbb{R})\left(w \in L^{\infty}(\mathbb{R})\right.$ for $p=2$ and $w \in L^{2}(\mathbb{R})$ for $\left.p=\infty\right)$. Then $w$ is an $L^{p}$-admissible majorant for $K_{\Theta}$ if and only if $H_{-}^{2} \oplus \Theta H^{2}$ is not dense in $L^{q}\left(w^{q}\right)$.

Let us single out the special case corresponding to the usual (i.e. $L^{\infty}$-) admissibility: a nonnegative $L^{2}$-function $w$ is in $\operatorname{Adm}(\Theta)$ if and only if $H_{-}^{2} \oplus \Theta H^{2}$ is not dense in $L^{1}(w)$.

The condition $w \in L^{2}(\mathbb{R})$ is not very restrictive, since it is clear that, for any majorant $w \in$ $\operatorname{Adm}(\Theta)$, there exists an admissible majorant $w_{1} \leqslant w$ a.e. on $\mathbb{R}$ such that $w_{1} \in L^{2}(\mathbb{R})$ (we set $w_{1}=|f|$, where $f \in K_{\Theta}$ is a nonzero function such that $\left.|f| \leqslant w\right)$.

Proof of Proposition 6.1. Consider the spaces $X=L^{2}(\mathbb{R}) \times L^{p}(\mathbb{R})$ and $Y=L^{2}(\mathbb{R}) \times L^{q}(\mathbb{R})$. Then $X=Y^{*}$ according to the usual identification with respect to the standard pairing

Set

$$
\left\langle\left(f_{1}, g_{1}\right),\left(f_{2}, g_{2}\right)\right\rangle=\int_{\mathbb{R}} f_{1} \bar{f}_{2}+g_{1} \bar{g}_{2} .
$$

$$
M=\left\{(f, f / w): f \in K_{\Theta}, f / w \in L^{p}(\mathbb{R})\right\} .
$$

Clearly, the majorant $w$ is not $L^{p}$-admissible if and only if $M=\{0\}$.

Consider now two subspaces of $Y$,

$$
\begin{aligned}
& S_{1}=\left\{(\psi,-\psi w): \psi \in L^{2}(\mathbb{R})\right\}, \\
& S_{2}=\left(L^{2}(\mathbb{R}) \ominus K_{\Theta}\right) \times\{0\}=\left(H_{-}^{2} \oplus \Theta H^{2}\right) \times\{0\} .
\end{aligned}
$$

The inclusion $S_{1} \subset Y$ follows from the relation $w \psi \in L^{q}(\mathbb{R})$ for any $\psi \in L^{2}(\mathbb{R})$, which, in turn, follows from the Hölder inequality with exponents $2 / q$ and $2 /(2-q)$ and the condition $w \in$ $L^{2 p /(p-2)}(\mathbb{R})$. The set $M$ can now be identified with the polar set (in $Y^{*}$ ) of $S=S_{1}+S_{2}$, and, by the Hahn-Banach theorem, $M=\{0\}$ if and only if $S$ is dense in $Y$. In other words, $w$ is not 
an $L^{p}$-admissible majorant for $K_{\Theta}$ if and only if, for any $f \in L^{2}(\mathbb{R}), g \in L^{q}(\mathbb{R})$, and $\varepsilon>0$, there exists a $\psi \in L^{2}(\mathbb{R})$ and an $h \in H_{-}^{2} \oplus \Theta H^{2}$ such that

$$
\|f-\psi-h\|_{2}+\|g+w \psi\|_{q}<\varepsilon \text {. }
$$

First, assume that $w$ is not $L^{p}$-admissible. Then, applying $(20)$ to $f=0$ and $g \in L^{q}(\mathbb{R})$, we see that $\|g+w \psi\|_{q}<\varepsilon$ for some $\psi \in H_{-}^{2} \oplus \Theta H^{2}$. Hence, $H_{-}^{2} \oplus \Theta H^{2}$ is dense in $L^{q}\left(w^{q}\right)$.

Conversely, assume that $H_{-}^{2} \oplus \Theta H^{2}$ is dense in $L^{q}\left(w^{q}\right)$. Let $f \in L^{2}(\mathbb{R})$ and let $f=f_{1}+f_{2}$, where $f_{1} \in K_{\Theta}$ and $f_{2} \in H_{-}^{2} \oplus \Theta H^{2}$. Set $\psi=f_{1}+\xi$ and $h=f_{2}-\xi$, where $\xi \in H_{-}^{2} \oplus \Theta H^{2}$ will be chosen below. Then the first norm in (20) is zero. Since $H_{-}^{2} \oplus \Theta H^{2}$ is dense in $L^{q}\left(w^{q}\right)$, we can choose a $\xi \in H_{-}^{2} \oplus \Theta H^{2}$ such that

$$
\left\|g+w f_{1}+w \xi\right\|_{q}<\varepsilon
$$

for given $g \in L^{q}(\mathbb{R})$ and $\varepsilon>0$. Hence, $w$ is not an $L^{p}$-admissible majorant.

\section{References}

[1] A. D. Baranov, "Polynomials in the de Branges spaces of entire functions," Ark. Mat., 44:1 (2006), 16-38.

[2] A. Baranov, "Completeness and Riesz bases of reproducing kernels in model subspaces," Internat. Math. Res. Notices, 2006 (to appear).

[3] A. D. Baranov, A. A. Borichev, and V. P. Havin, "Majorants of meromorphic functions with fixed poles," Indiana Univ. Math. J. (to appear).

[4] A. Beurling and P. Malliavin, "On Fourier transforms of measures with compact support," Acta Math., 107 (1962), 291-309.

[5] L. de Branges, Hilbert Spaces of Entire Functions, Prentice-Hall, Englewood Cliffs, NJ, 1968.

[6] J. A. Cima and W. T. Ross, The Backward Shift on the Hardy Space, Math. Surveys Monographs, vol. 79, Amer. Math. Soc., Providence, RI, 2000.

[7] V. Havin and B. Jöricke, The Uncertainty Principle in Harmonic Analysis, Springer-Verlag, Berlin, 1994.

[8] V. P. Havin and J. Mashreghi, "Admissible majorants for model subspaces of $H^{2}$. Part I: slow winding of the generating inner function," Canad. J. Math., 55:6 (2003), 1231-1263.

[9] V. P. Havin and J. Mashreghi, "Admissible majorants for model subspaces of $H^{2}$. Part II: fast winding of the generating inner function," Canad. J. Math., 55:6 (2003), 1264-1301.

[10] M. Kaltenbäck and H. Woracek, "Hermite-Biehler functions with zeros close to the imaginary axis," Proc. Amer. Math. Soc., 133:1 (2005), 245-255.

[11] P. Koosis, The Logarithmic Integral I, Cambridge Stud. Adv. Math., vol. 12, Cambridge University Press, Cambridge, 1988.

[12] P. Koosis, The Logarithmic Integral II, Cambridge Stud. Adv. Math., vol. 21, Cambridge University Press, Cambridge, 1992.

[13] P. Koosis, Leçons sur le théorème de Beurling et Malliavin, Les Publications CRM, Montréal, 1996.

[14] J. Mashreghi, F. L. Nazarov, and V. P. Havin, "The Beurling-Malliavin multiplier theorem: the seventh proof," Algebra i Analiz, 17:5 (2005), 3-68.

[15] H. P. McKean, "Some questions about Hardy functions," in: Linear and Complex Analysis, Problem Book 3, Part I, Lecture Notes in Math., vol. 1573 (eds. V. P. Havin, N. K. Nikolski), 1994, 157-158.

[16] N. K. Nikolski, Operators, Functions, and Systems: an Easy Reading. Vol. 1-2, Math. Surveys Monographs, vol. 92-93, Amer. Math. Soc., Providence, RI, 2002.

[17] V. V. Peller, Hankel Operators and Their Applications, Springer-Verlag, 2003.

S.-Petersburg State University

e-mail: havin@VH1621.spb.edu

S.-Petersburg State University

e-mail: antonbaranov@netscape.net

Translated by A. D. Baranov and V. P. Havin 